\title{
Energy Spectra of tightly Confined Systems
}

\author{
Ahmed Al-Jamel ${ }^{1,2}$ \\ ${ }^{1}$ Department of Mathematics and Physical Sciences, College of Arts and Sciences, University of Nizwa, Nizwa, \\ Sultanate of Oman \\ ${ }^{2}$ Physics Department, Al Al-Bayt University, Mafraq 25113, Jordan \\ Correspondence: Ahmed Al-Jamel, Department of Mathematics and Physical Sciences, College of Arts and \\ Sciences, University of Nizwa, Nizwa, Sultanate of Oman. E-mail: aaljamel@gmail.com
}

Received: August 12, 2015 Accepted: August 25, 2015 Online Published: September 5, 2015

doi:10.5539/apr.v7n5p80 URL: http://dx.doi.org/10.5539/apr.v7n5p80

\begin{abstract}
An approximate recipe to the energy eigenvalues for the problem of non-relativistic hydrogen-like atom confined in a spherical cavity of impenetrable wall is presented. The method is based on proposing an ansatz solution with a cut-off function. The asymptotic behavior of the reduced Schrödinger equation is then considered for large $r$ and small $r$. Some approximations are applied to deform some terms of the equation, which then allowed us to apply the Nikiforov-Uvarov method in both regions. A potential parameter $\delta$ is introduced that plays a crucial role in the calculations. In both cases, we obtained an algebraic equation for the energy, which then can be solved for the specific potential parameters.
\end{abstract}

Keywords: confined systems, energy spectra, Schrödinger, Nikiforov-Uvarov

\section{Introduction}

There is a considerable interest in the development of the idea of a quantum system confined by a spherical potential. When such a system is trapped within a microscopic cavity, or is subjected to a high pressure, its atomic energy spectrum changes substantially as compared to its free counterparts (Sen, Garza, Vargas, \& Aquino, 2002; Xie, 2009), and thus it is of considerable interest to investigate it. Historically, the idea of such confined quantum system was proposed by Michels, de Boer and Bijl (1937) to simulate the variation of the static dipole polarizability of a confined hydrogen atom under very high pressures. Since then, such systems attracted considerable attention, and became a popular topic. There are many other quantum systems that experience confinement, and, in general, these confinement phenomena, extended from quark systems to molecules, are described by certain potential models; see for examples (Al-Jamel \& Widyan, 2012; Hall, Nasser, \& Sen, 2011; Boroun \& Abdolmalki, 2009; Hassanabadi \& Rajabi, 2009; Zalewski, 1998; Ding, Li, \& Shen, 1999) and many others.

One of the main obstacles in investigating such systems is the absence of the exact solutions of Schrödinger equation for such systems under Dirichlet-like boundary conditions on the wavefunctions imposed at the surface of the cavity, particularly for the cases where other than the s-wave are of interest. For such cases, there are some analytical and numerical approximation methods such as $1 / N$ expansion (Bag, Panja, \& Dutt, 1992), supersymmetry (Morales, 2004), Pekeris approximation (Pekeris, 1934), variational methods (Montgomery, 2001, 2011), and asymptotic iteration methods (Ciftci, Hall, \& Saad, 2009) to obtain the energy eigenvalues and eigenfunctions with this type of boundary conditions. Another technique used to find exact solutions of quantum systems is the Nikiforov-Uvarov method (Nikiforov \& Uvarov, 1988; Szego, 1975), which is now used often but for non confined systems by many authors.For detailed applications and applicability of the Nikiforov-Uvarov method in quantum mechanics, the reader may refer to (Berkdemir, 2012).

In the present paper, we attempt to obtain the energy spectrum of the problem of a tightly confined atom using Nikiforov-Uvarov method. In section 2, we review the Nikiforov-Uvarov (NU) technique. In section 3, we describe the problem, and the asymptotic form of the Schrödinger equation that allows us to apply the NU method. In section 4. results and discussion are given. In the last section, summary and conclusions are presented. In what follows, we adopt the the atomic (or Hartree) units $\hbar=c=m=e=1$, where $m$ and $e$ are the electron mass and charge, respectively. 


\section{Nikiforov-Uvarov Method}

The Nikiforov-Uvarov method (hereafter, NU) has been used by many authors to find the energy eigenvalues and eigenfunctions of Schrödinger-like equation, for certain potentials of interest. It is based on finding a suitable coordinate transformation $s=s(x)$ to convert a general second order linear differential equation, with special orthogonal functions, into generalized hypergeometric equation of the main form (Nikiforov \& Uvarov, 1988; Szego, 1975; Berkdemir, 2012)

$$
\psi^{\prime \prime}(s)+\frac{\tilde{\tau}(s)}{\sigma(s)} \psi^{\prime}(s)+\frac{\widetilde{\sigma}(s)}{\sigma^{2}(s)} \psi(s)=0
$$

where $\sigma(s)$ and $\widetilde{\sigma}(s)$ are polynomials, at most second-degree, and $\tilde{\tau}(s)$ is a first-degree polynomial. With the following transformation

$$
\psi(s)=\phi(s) y(s),
$$

Equation (1) is reduced to an equation of hypergeometric type

$$
\sigma(s) y^{\prime \prime}+\tau(s) y^{\prime}+\lambda y=0,
$$

where $\phi(s)$ satisfies

$$
\phi(s)^{\prime} / \phi(s)=\pi(s) / \sigma(s),
$$

and $y(s)$ is the hypergeometric type function whose polynomial solutions can be generated by the Rodriguez formula

$$
y_{n}(s)=\frac{B_{n}}{\rho(s)} \frac{d^{n}}{d s^{n}}\left[\sigma^{n}(s) \rho(s)\right],
$$

where $B_{n}$ is the normalization factor and $\rho$ is called the weight function and it satisfies the condition

$$
(\sigma \rho)^{\prime}=\tau \rho .
$$

The function $\pi$ and the parameter $\lambda$ in this algorithm are defined as

$$
\pi=\frac{\sigma^{\prime}-\tilde{\tau}}{2} \pm \sqrt{\left(\frac{\sigma^{\prime}-\tilde{\tau}}{2}\right)^{2}-\widetilde{\sigma}+k \sigma}
$$

and

$$
\lambda=k+\pi^{\prime} .
$$

Here, $\pi(s)$ is a polynomial with the parameter $s$. The determination of $k$ plays a crucial role in the calculation of $\pi(s)$. To determine the applicable values of $k$, the expression under the square root in Equation (7) must be square of a polynomial. Hence, the following new eigenvalue equation for the Schrödinger equation will be established

$$
\lambda=\lambda_{n}=-n \tau^{\prime}-\frac{n(n-1)}{2} \sigma^{\prime \prime},(n=0,1,2, \ldots)
$$

where

$$
\tau(s)=\tilde{\tau}(s)+2 \pi(s)
$$

and it will have a negative derivative. By comparing Equation (8) with Equation (9), we obtain the energy eigenvalues.

\section{Eigenvalues and Eigenfunctions}

For concreteness, we restrict ourselves with the confined H-atom system. Consider a hydrogen-like atom that is enclosed in an impenetrable shell such as the nucleus resides at the center and its electron is subject to a potential that is infinite in all space except within a spherical cavity of radius $r_{0}$

$$
V(r)= \begin{cases}-\frac{z}{r}, & \text { if } 0<r<r_{0} \\ \infty, & \text { elsewhere }\end{cases}
$$

where $Z$ is a constant. In prinicple, the system can also be of two-electrons confined within the cavity but with $Z<0$. The radial Schrödinger equation for this system is 


$$
\frac{d^{2} R}{d r^{2}}+\frac{2}{r} \frac{d R}{d r}+2\left(E+\frac{Z}{r}-\frac{\gamma}{2 r^{2}}\right) R(r)=0
$$

subject to Dirichlet boundary condition $R\left(r_{0}\right)=0$, where $\gamma=l(l+1)$. We usually use the ansatz $R(r)=$ $\frac{1}{r} u(r)$ for solving central problems. Here we propose instead an ansatz of the form

$$
R(r)=\frac{1}{r}\left(1-\frac{r}{r_{0}}\right) \chi(r)
$$

such as

$$
\lim _{r \rightarrow 0} \frac{\chi(r)}{r}=0,
$$

which means that $\chi(r)$ is vanishing faster that $r$ as approaching the origin. The factor $\left(1-\frac{r}{r_{0}}\right)$ is a cut-off function, which is included to ensure that the wave function satisfies the boundary condition. Substituting this into Equation (12) we obtain after some algebra that $\chi(r)$ satisfies:

$$
\chi^{\prime \prime}(r)-\frac{2}{r_{0}-r} \chi^{\prime}(r)+\frac{-\gamma+2 Z r+2 E r^{2}}{r^{2}} \chi(r)=0
$$

which can be re-casted on the form

$$
\chi^{\prime \prime}(r)-\frac{2}{\left(r_{0}-r\right)} \chi^{\prime}(r)+\frac{Q(r)}{\left(r_{0}-r\right)^{2}} \chi(r)=0
$$

where,

$$
Q(r) \equiv-\gamma-4 Z r_{0}+2 r_{0}^{2} E-\frac{\gamma r_{0}^{2}}{r^{2}}+\frac{2 r_{0}\left(Z r_{0}+\gamma\right)}{r}+\left(2 Z-4 r_{0} E\right) r+2 E r^{2} .
$$

At this moment, we divide the cavity into two sub-regions: large $\mathrm{r}$, and small $\mathrm{r}$, and then consider the behavior of the solution in each sub-region. Our main goal here is to approximate $Q(r)$ by a quadratic function so that we can apply NU method.

\subsection{Asymptotic Solution at Large $\boldsymbol{r}\left(\boldsymbol{r} \sim \boldsymbol{r}_{\mathbf{0}}\right)$}

At large r, closer to the shell, we apply an approximation scheme, similar to the so-called Pekeris approximation,

on the two terms $\frac{1}{r^{2}}$ and $\frac{1}{r}$. It is based on the expansion of such terms in a power series about some point within the cavity $0<\delta<r_{0}$ up to the second order. This helps to deform the function $Q(r)$ so that it becomes quadratic in $r$ and thus the resulted differential equation can be treated by NU technique. Setting $r=(x+\delta)$, and around $x=0$ these two terms can be expanded into a power series as

$$
\frac{2 r_{0}\left(Z r_{0}+\gamma\right)}{(x+\delta)}-\frac{\gamma r_{0}^{2}}{(x+\delta)^{2}} \approx-\frac{\left(-2 Z \delta r_{0}^{2}+\gamma r_{0}^{2}-2 \gamma \delta r_{0}\right)}{\delta^{2}}-\frac{2\left(z \delta r_{0}^{2}-\gamma r_{0}^{2}+\gamma \delta r_{0}\right)}{\delta^{3}} x+\frac{\left(2 Z \delta r_{0}^{2}-3 \gamma r_{0}^{2}+2 \gamma \delta r_{0}\right)}{\delta^{4}} x^{2} .
$$

Within this approximation, both sides of Equation (18) were plotted for some values of the parameters $Z, r_{0}, l$ and $\delta$, and then compared with the exact form, and it is found that the expansion is reasonable by controlling the value of $\delta$; see Figure 1. This parameter $\delta$ plays a crucial rule in the calculations as we shall see in the discussion of the results. The function $Q(r)$ then becomes

$$
Q(r) \approx A+B r+C r^{2}
$$

where

$$
\begin{gathered}
A=\frac{6 r_{0}\left(Z \delta r_{0}+\gamma \delta-\gamma r_{0}\right)}{\delta^{2}}-\left(4 Z r_{0}+\gamma-2 r_{0}^{2} E\right) \\
B=-\frac{r_{0}\left(6 Z \delta r_{0}+3 \gamma \delta-8 \gamma r_{0}\right)}{\delta^{3}}+2 Z-4 r_{0} E \\
C=2 E+\frac{r_{0}\left(2 Z \delta r_{0}+2 \gamma \delta-3 \gamma r_{0}\right)}{\delta^{4}}
\end{gathered}
$$


Therefore, Equation (16) becomes

$$
\chi^{\prime \prime}(r)-\frac{2}{\left(r_{0}-r\right)} \chi^{\prime}(r)+\frac{A+B r+C r^{2}}{\left(r_{0}-r\right)^{2}} \chi(r)=0
$$
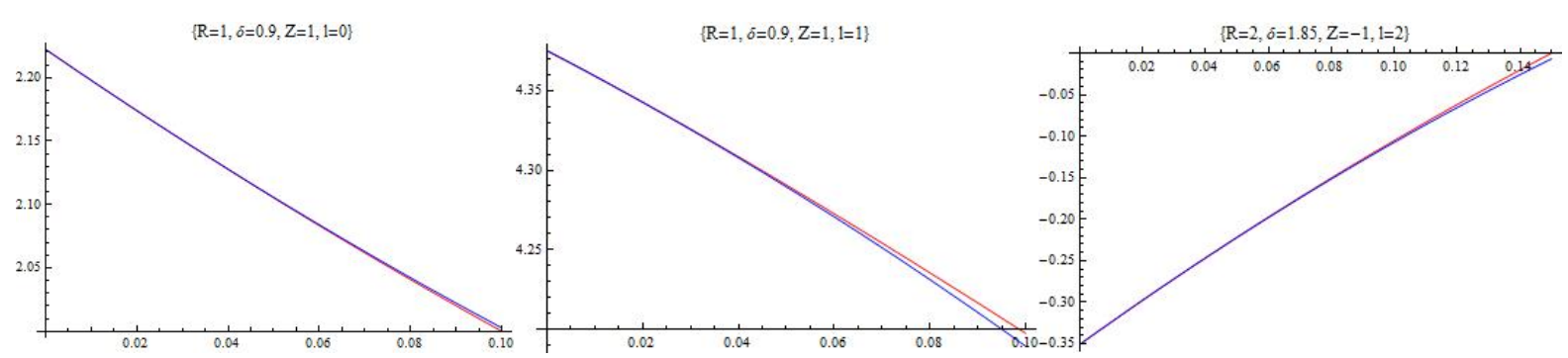

Figure 1. The approximation of Equation (18) for different set of values of $Z, l, r_{0}$ and $\delta$

For the NU-method, we have now, $\tilde{\tau}=-2, \sigma=\left(r_{0}-r\right), \widetilde{\sigma}=A+B r+C r^{2}$. Then

$$
\pi=\frac{1}{2} \pm \sqrt{\frac{1}{4}-A-B r-C r^{2}+k\left(r_{0}-r\right)} .
$$

The constant $k$ is chosen such as the discriminant of function under the square is zero, i.e. $\Delta=(k+B)^{2}+$ $4 C\left(\frac{1}{4}-A+k r_{0}\right)=0$. Therefore,

$$
\begin{gathered}
k_{ \pm}=-B-2 C r_{0} \pm \sqrt{-C+4 C^{2} r_{0}^{2}+4 A C+4 B C r_{0}} \\
\pi=\frac{1}{2} \pm \frac{1}{2}\left(2 \sqrt{-C} r+2 C r_{0} \mp \sqrt{-C+4 C^{2} r_{0}^{2}+4 A C+4 B C r_{0}}\right) .
\end{gathered}
$$

Thus,

$$
\tau=-2+2 \pi
$$

For physically bound state solutions, we choose the positive sign in the above equation so that the derivative

$$
\tau^{\prime}=-2 \sqrt{-C}
$$

can have negative values depending on the parameters $Z, l, r_{0}$ and $\delta$. Using Equation (8), then we have

$$
\lambda=-B-2 C r_{0} \pm \sqrt{-C-4 C^{2} r_{0}^{2}+4 A C+4 B C r_{0}}-\sqrt{-C}
$$

On the other hand, from Equation (9)

$$
\lambda_{n}=2 n \sqrt{-C}
$$

Comparing Equation (28) and Equation (29), the following algebraic equation for energy at large $r$ is obtained

3.2 Asymptotic Solution at Small $r(\boldsymbol{r}<<\boldsymbol{R})$

$$
-B-2 C r_{0} \pm \sqrt{-C+4 C^{2} r_{0}^{2}+4 A C+4 B C r_{0}}=(2 n+1) \sqrt{-C} .
$$

At small $r$, we can ignore the linear and quadratic terms in $r$, and then we have

$$
Q(r) \approx-2 \gamma-4 a r_{0}+2 r_{0}^{2} E-\frac{\gamma r_{0}^{2}}{r^{2}}+\frac{2 r_{0}\left(a r_{0}+\gamma\right)}{r}
$$

which can be rewritten as

$$
Q(r) \approx a+\frac{b}{r}+\frac{c}{r^{2}}=\frac{1}{r^{2}}\left(a r^{2}+b r+c\right)
$$

where

$$
\begin{gathered}
a=-\gamma-4 Z r_{0}+2 r_{0}^{2} E \\
b=2 r_{0}\left(Z r_{0}+\gamma\right) \\
c=-\gamma r_{0}^{2}
\end{gathered}
$$


Therefore, Equation (17) becomes

$$
\chi^{\prime \prime}(r)-\frac{2 r}{r\left(r_{0}-r\right)} \chi^{\prime}(r)+\frac{\left(a r^{2}+b r+c\right)}{r^{2}\left(r_{0}-r\right)^{2}} \chi(r)=0
$$

For the NU-method, we have now, $\tilde{\tau}=-2 r, \sigma=r\left(r_{0}-r\right), \widetilde{\sigma}=a r^{2}+b r+c$. Then

$$
\pi=\frac{r_{0}}{2} \pm \sqrt{\frac{r_{0}^{2}}{4}-a r^{2}-b r-c+k r\left(r_{0}-r\right)} .
$$

The constant $k$ is chosen such as the discriminant of function under the square is zero, $\Delta=\left(k r_{0}-b\right)^{2}+$ $4(a+k)\left(\frac{r_{0}^{2}}{4}-c\right)=0$. Therefore,

$$
k_{ \pm}=\frac{1}{2 r_{0}^{2}}\left(2 b r_{0}+4 c-r_{0}^{2} \pm \sqrt{4 c-r_{0}^{2}} \sqrt{4 a r_{0}^{2}+4 b r_{0}+4 c-r_{0}^{2}}\right)
$$

Thus,

$$
\pi=\frac{r_{0}}{2} \pm\left(\sqrt{-(a+k)} r+\sqrt{\frac{r_{0}^{2}}{4}-c}\right)
$$

Thus,

$$
\tau=-2+2 \pi
$$

For physically bound state solutions, we choose the positive sign in the above equation so that the derivative

$$
\tau^{\prime}=-2-2 \sqrt{-(a+k)}
$$

can have negative values. Using Equation (8), then we have

$$
\lambda=k-\sqrt{-(a+k)}
$$

On the other hand, from Equation (9)

$$
\lambda_{n}=2 n+2 n \sqrt{-(a+k)}+n(n+1)
$$

Comparing Equation (41) and Equation (42), the following algebraic equation for energy at small $r$ is obtained

\section{Results and Discussion}

$$
n^{2}+n+(2 n+1) \sqrt{-(a+k)}=k \text {. }
$$

In this section, we employ the results of the above analysis to obtain the energy eigenvalues of the confined atom for certain values of $r_{0}$ and $Z$. To find the average total energy eigenvalues of the system, the results obtained from small distances and large distances are combined together

$$
<E>=E_{\text {small }}+E_{\text {large }}
$$

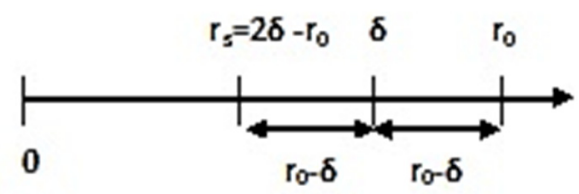

Figure 2. The parameters $\delta$ and $r_{s}$ and the relation between them in terms of $r_{0}$

where $E_{\text {large }}$ is given from Equation (30) while $E_{\text {small }}$ is given from Equation (43). This may be understood as follows: The expectation value of the energy is:

$$
<E>=\int_{0}^{s} \psi^{*} H \psi d^{3} \vec{r}+\int_{s}^{r_{0}} \psi^{*} H \psi d^{3} \vec{r}
$$

where $s$ is the separation surface between small region and large region. To apply the recipe described in this work, we firstly divide the cavity into two sub-regions $\left[0, r_{s}\right]$ and $\left[r_{s}, r_{0}\right]$. Then we use Equation (30) to obtain the energy $E_{\text {large }}$ by using a suitable value for $\delta$ that fits Equation (18). The value of the separation radius $r_{s}$ can then be determined from $r_{s}=2 \delta-r_{0}$; see Figure 2. The values of $r_{0}$ and $\delta$ can be plugged in Equation (30) along with the other constants to determine $E_{\text {large }}$, while $r_{s}$ is used in Equation (43) to obtain $E_{\text {small }}$ 
different values of $r_{0}$. It should be mentioned that a special care of the choice of $\delta$ and potential parameters is demanded to obtain real energy spectra.

One of the interest quantities to calculate is the charge radius $r_{q}$, which is the quadratic mean value of $r$ :

$$
r_{q}=\sqrt{\left\langle r^{2}>\right.}=\int_{0}^{s} \psi^{*} r^{2} \psi d^{3} \vec{r}+\int_{s}^{r_{0}} \psi^{*} r^{2} \psi d^{3} \vec{r} .
$$

This quantity is related to the electric polarizability of the system. For a certain set of potential parameters, one can in principle obtain the NU functions needed to construct the wavefunction in both regions and then calculate various properties such as $r_{q}$.

As an application, we consider the s-wave $(l=0, n=0)$ energy eigenvalue and charge radius. Taking $Z=1$ and $r_{0}=0.1$, and with the fitting choise $\delta=0.08$ then $r_{s}=0.06$. Therefore using Equation (30) and Equation (43), we obtain $\langle E\rangle=105.1535$ Hartrees, which is equivalent to $201.3070 \mathrm{Ry}$. The corresponding exact value is 937.986 Ry (Varshni, 1997). The difference between the two results indicates that the intermediate region should be considered more carefully, which will be our proposal of future work.

\section{Summary and Conclusions}

In this paper, we describe a recipe to find the energy eigenvalues of a confined $\mathrm{H}$-like atom for any $l$-value and subject to the condition of confinement within a finite radius $r_{0}$. An ansatz that takes into account the cut off at the surface is introduced. The obtained differential equation was solved by considering two regions: small $r$ and large $r$. The UV method was then used to obtain expressions for the energy eigenvalues. The method may be extended to potentials that contains also linear and/or harmonic oscillator terms, and even to two-electron systems. We hope this method is of interest to the nanostructure and quantum dots community.

\section{Acknowledgments}

This work is performed during a sabbatical leave 2014-2015 provided by Al al-Bayt University in Jordan to Dr. Ahmed Al-Jamel which was spent at University of Nizwa, Sultanate of Oman. I thank everybody who provided fruitful comments.

\section{References}

Al-Jamel, A., \& Widyan, H. (2012). Heavy Quarkonium Mass Spectra in A Coulomb Field Plus Quadratic Potential Using Nikiforov-Uvarov Method. Applied Physics Research, 4(3). http://dx.doi.org/10.5539/apr. v4n3p94

Bag, M., Panja, M. M., \& Dutt, R. (1992). Modified shifted large- $N$ approach to the Morse oscillator. Phys. Rev. A, 46, 9. http://d x.doi.org/10.1103/PhysRevA.46.6059

Berkdemir, C. (2012). Application of the Nikiforov-Uvarov Method in Quantum Mechanics. In M. R. Pahlavani (Ed.), Theoretical Concepts of Quantum Mechanics. http://dx.doi.org/10.5772/33510

Boroun, G. R., \& Abdolmalki, H. (2009). Variational and exact solutions of the wavefunction at origin (WFO) for heavy quarkonium by using a global potential. Physica Scripta, 80(6), 065003. http://dx.doi.org/10. 1088/0031-8949/80/06/065003

Ciftci, H., Hall, R. L., \& Saad, N. (2009). Study of a confined hydrogen-like atom by the asymptotic iteration method. International Journal of Quantum Chemistry, 109, 931-93. http://dx.doi.org/10.1002/qua.21905

Ding, Y. B., Li, X. Q., \& Shen, P. N. (1999). Variational estimation of the wave function at the origin for heavy quarkonium. Physical Review D, 60(7), 074010. http://dx.doi.org/10.1103/PhysRevD.60.074010.

Hall, R. L. , Nasser, H., \& Sen, K. D. (2011). Spectral characteristics for a spherically confiined $-\mathrm{a} / \mathrm{r}+\mathrm{br}^{2}$ potential. J. Phys. A: Math. Theor., 44-185307. http://dx.doi.org/10.1088/1751-8113/44/18/185307

Hassanabadi, H., \& Rajabi, A. A. (2009). Energy levels of a spherical quantum dot in a confining potential. Physics Letters A, 373(6), 679-681. http://dx.doi.org/10.1016/j.physleta.2008.12.038

Michels. A., de Boer. J., \& Bijl, A. (1937). Remarks concerning molecular interaction and their influence on the polarizability. Physica, 4, 981-994.

Montgomery, H. E., Jr. (2001). Variational Perturbation Theory of the Confined Hydrogen Atom. Int. J. Mol. Sci., 2, 103-108. http://dx.doi.org/10.3390/i2020103

Montgomery, H. E., Jr. (2011). Variational perturbation treatment of the confined hydrogen atom. Eur. J. Phys., 32, 1275. http://dx.doi.org/10.1088/0143-0807/32/5/015 
Morales, D. A. (2004). Supersymmetric improvement of the Pekeris approximation for the rotating Morse potential. Chem. Phys. Letters, 394, 68. http://dx.doi.org/10.1016/j.cplett.2004.06.109

Nikiforov, A. F., \& Uvarov, V. B. (1988). Special Functions of Mathematical Physics (Birkhauser, Basel).

Pekeris, C. L. (1934). The Rotation-Vibration Coupling in Diatomic Molecules. Phys. Rev., 45, 98. http://dx.doi.org/10.1103/PhysRev.45.98

Sen, K. D., Garza, J., Vargas, R., \& Aquino, N. (2002). Static dipole polarizability of shell-confined hydrogen atom. Physics Letters A, 295(5), 299-304. http://dx.doi.org/10.1016/S0375-9601(02)00148-2

Szego, G. (1975). Orthogonal Polynomials (4th ed.). American Mathematical Society.

Varshni, Y. P. (1997). Accuratewavefunctions for the confined hydrogen atom at high pressures.Journal of physics. B, Atomic molecular and optical physics, 30(18), 589-593.

Xie, W. (2009). Effects of an electric field on the confined hydrogen atom in a parabolic potential well. Physics Letters A, 373(26), 2251-2254. http://dx.doi.org/10.1016/j.physleta.2009.04.058

Zalewski, K. (1998). Nonrelativistic Description of Heavy Quarkonia. Acta Phys. Polon. B, 29, 2535-2538.

\section{Copyrights}

Copyright for this article is retained by the author(s), with first publication rights granted to the journal.

This is an open-access article distributed under the terms and conditions of the Creative Commons Attribution license (http://creativecommons.org/licenses/by/3.0/). 